\title{
Existence of solutions for Schrödinger-Poisson system with asymptotically periodic terms
}

\section{Da-Bin Wang*, Lu-Ping Ma, Wen Guan, Hong-Mei Wu}

Department of Applied Mathematics, Lanzhou University of Technology, 730050 Lanzhou, People's Republic of China.

\author{
Communicated by V. K. Le
}

\section{Abstract}

In this paper, we consider the following nonlinear Schrödinger-Poisson system

$$
\begin{cases}-\Delta u+V(x) u+K(x) \phi u=f(x, u), & x \in \mathbb{R}^{3}, \\ -\Delta \phi=K(x) u^{2}, & x \in \mathbb{R}^{3},\end{cases}
$$

where $\mathrm{V}, \mathrm{K} \in \mathrm{L}^{\infty}\left(\mathbb{R}^{3}\right)$ and $\mathrm{f}: \mathbb{R}^{3} \times \mathbb{R} \rightarrow \mathbb{R}$ is continuous. We prove that the problem has a nontrivial solution under asymptotically periodic case of $\mathrm{V}, \mathrm{K}$, and $\mathrm{f}$ at infinity. Moreover, the nonlinear term $\mathrm{f}$ does not satisfy any monotone condition.

Keywords: Schrödinger-Poisson system, asymptotically periodic, variational method.

2010 MSC: 34C25, 58E50.

(C)2018 All rights reserved.

\section{Introduction and main result}

For past decades, much attention has been paid to the nonlinear Schrödinger-Poisson system

$$
\begin{cases}i \hbar \frac{\partial \Psi}{\partial t}=-\frac{\hbar^{2}}{2 m} \Delta \Psi+\phi(x) \Psi-|\Psi|^{q-1} \Psi, & x \in \mathbb{R}^{3}, t \in \mathbb{R}, \\ -\Delta \phi=|\Psi|^{2}, & x \in \mathbb{R}^{3},\end{cases}
$$

where $\hbar$ is the Planck constant. System (1.1) derived from quantum mechanics. For this system, the existence of stationary wave solutions is often sought, that is, the following form of solutions

$$
\Psi(x, t)=e^{i t} u(x), x \in \mathbb{R}^{3}, t \in R .
$$

\footnotetext{
${ }^{*}$ Corresponding author

Email addresses: wangdb96@163.com (Da-Bin Wang), 974531947@qq.com (Lu-Ping Ma), mathguanw@163.com (Wen Guan), wuhongmei0610@126.com (Hong-Mei Wu)

doi: 10.22436/jnsa.011.05.01
}

Received: 2017-01-29 Revised: 2017-11-22 Accepted: 2018-01-11 
Therefore, the existence of the standing wave solutions of the system (1.1) is equivalent to finding the solutions of the following system

$$
\begin{cases}-\frac{\hbar^{2}}{2 m} \Delta u+\hbar u+\phi u=|u|^{q-1} u, & x \in \mathbb{R}^{3}, \\ -\Delta \phi=u^{2}, & x \in \mathbb{R}^{3} .\end{cases}
$$

Let $m=\frac{1}{2}$ and $\hbar=1$, system (1.2) becomes the following system

$$
\begin{cases}-\Delta u+u+\phi u=|u|^{q-1} u, & x \in \mathbb{R}^{3}, \\ -\Delta \phi=u^{2}, & x \in \mathbb{R}^{3} .\end{cases}
$$

There was a series of work to discuss the existence, non existence, radially symmetric solutions, non-radially symmetry solutions, ground states, semiclassical states and sign-changing solutions to Schrödinger-Poisson system (1.3) by using the variational method $[1,2,5-7,9-13,17-19,21-24,28,29,32$, $34,37,38,40-42,44-46]$.

In case $3<q<5$, Coclite [10] considered the nontrivial radially symmetric solutions for system (1.3). In [11], when $3 \leqslant q<5$, D'Aprile and Mugnai obtained similar results. By using Pohozaev's identity, in [12], D'Aprile and Mugnai considered the non existence of nontrivial solution to system (1.3) in case $q \leqslant 1$ or $q \geqslant 5$.

In [32], Ruiz studied the following Schrödinger-Poisson system

$$
\begin{cases}-\Delta u+u+\lambda \phi u=u^{p}, & x \in \mathbb{R}^{3}, \\ -\Delta \phi=u^{2}, & x \in \mathbb{R}^{3},\end{cases}
$$

where $\lambda>0$ is parameter and $1<p<5$. Using the mountain pass theorem and Ekeland variational principle, Ruiz proved that system (1.4) has at least two (one) positive radial solutions when $1<p<2$ $(p=2)$ and $\lambda>0$ sufficiently small and system (1.4) has no nontrival solution when $1<p \leqslant 2$ and $\lambda \geqslant \frac{1}{4}$. Moreover, by applying the method of finding the minimal sequence on a manifold associated with the Nehari manifold and the Pohozaev's identity, Ruiz proved that the system (1.4) has a positive radial solution in case $2<p<5$.

In [5], Ambrosetti and Ruiz obtained the existence of infinitely many radially symmetric solutions to system (1.4) when $2<p \leqslant 5$.

Using Lyapunov-Schmidt reduction method, D'Aprile and Wei [13] obtained the bound state solution for system (1.3), and the concentration of the solution is also studied. With regard to other relevant results, please see $[23,24,40]$.

In [2], Alves et al. studied Schrödinger-Poisson system

$$
\begin{cases}-\Delta u+V(x) u+\phi u=f(u), & x \in \mathbb{R}^{3}, \\ -\Delta \phi=u^{2}, & x \in \mathbb{R}^{3},\end{cases}
$$

where $\mathrm{V}$ is bounded, local Hölder continuous, and satisfies:

(1) $V(x) \geqslant \alpha>0, x \in \mathbb{R}^{3}$;

(2) $\mathrm{V}(\mathrm{x})=\mathrm{V}(\mathrm{x}+\mathrm{y}), \forall \mathrm{x} \in \mathbb{R}^{3}, \forall y \in \mathbb{Z}^{3}$;

(3) $\lim _{|x| \rightarrow \infty}\left|V(x)-V_{0}(x)\right|=0$;

(4) $V(x) \leqslant V_{0}(x), \forall x \in \mathbb{R}^{3}$, and there exists $\Omega \subset \mathbb{R}^{3}$ such that

$$
\mathrm{V}(\mathrm{x}) \leqslant \mathrm{V}_{0}(\mathrm{x}), \forall x \in \Omega
$$

where $V_{0}$ satisfies (2).

Alves studied the ground sates solutions to system (1.5) in case the periodic condition under (1)-(2) and in case the asymptotically periodic condition under (1), (3), and (4), respectively. 
In case $p \in(3,5)$, Cerami and Vaira [9] studied the existence of positive solutions for the following non-autonomous Schrödinger-Poisson system

$$
\begin{cases}-\Delta u+u+K(x) \phi(x) u=a(x)|u|^{p-1} u, & x \in \mathbb{R}^{3}, \\ -\Delta \phi=K(x) u^{2}, & x \in \mathbb{R}^{3},\end{cases}
$$

where $a, K$ are nonnegative functions such that $\lim _{|x| \rightarrow \infty} a(x)=a_{\infty}>0, \lim _{|x| \rightarrow \infty} K(x)=0$.

In [45], Zhang et al. studied existence of positive ground state solutions for the following SchrödingerPoisson system

$$
\begin{cases}-\Delta u+V(x) u+K(x) \phi u=f(x, u), & x \in \mathbb{R}^{3}, \\ -\Delta \phi=K(x) u^{2}, & x \in \mathbb{R}^{3} .\end{cases}
$$

where $\mathrm{V}, \mathrm{K}$, and $\mathrm{f}$ are asymptotically periodic at infinity. Moreover, the nonlinear term $\mathrm{f}$ satisfies the monotone condition: $\forall \mathrm{t} \neq 0, \mathrm{~s} \mapsto \frac{\mathrm{f}(\mathrm{x}, \mathrm{st}) \mathrm{t}}{\mathrm{s}^{3}}$ is nondecreasing on $(0, \infty)$.

On the other hand, when $K=0$, the Schrödinger-Poisson equation (1.7) becomes the standard Schrödinger equation (replace $\mathbb{R}^{3}$ with $\mathbb{R}^{\mathrm{N}}$ )

$$
-\Delta u+V(x) u=f(x, u), \quad x \in \mathbb{R}^{N} .
$$

The Schrödinger equation (1.8) has been widely investigated by many authors in the last decades, see [3, $8,14-16,20,26,30,31]$ and reference thein.

Especially, in [14], Marchi studied the nontrivial solutions and ground state solutions for problem (1.8) in which $\mathrm{V}, \mathrm{f}$ satisfies the asymptotic periodic condition. In the context about asymptotic periodic, we refer the reader to $[25,27,35,36]$.

Motivated by above results, especially by $[2,14,45]$, in this paper we study nontrivial solutions and ground state solutions to system (1.7) under asymptotically periodic case of $V, K$, and $f$ at infinity.

Let $\mathfrak{I}$ be the functions $h \in \mathrm{L}^{\infty}\left(\mathbb{R}^{3}, \mathbb{R}\right)$ such that, for every $\varepsilon>0$, the set $\left\{x \in \mathbb{R}^{3}:|h(x)| \geqslant \varepsilon\right\}$ has finite Lebesgue measure. To state our main result, we assume that:

$\left(\mathrm{H}_{1}\right) \mathrm{V}, \mathrm{K} \in \mathrm{L}^{\infty}\left(\mathbb{R}^{3}\right), \inf _{x \in \mathbb{R}^{3}} \mathrm{~V}(\mathrm{x})>0, \inf _{\mathrm{x} \in \mathbb{R}^{3}} \mathrm{~K}(\mathrm{x})>0$;

$\left(\mathrm{H}_{2}\right) \mathrm{f} \in \mathrm{C}\left(\mathbb{R}^{3} \times \mathbb{R}, \mathbb{R}\right),|\mathrm{f}(\mathrm{x}, \mathrm{u})| \leqslant \mathrm{C}\left(1+|\mathrm{u}|^{\mathrm{p}}\right), 3<\mathrm{p}<5$;

$\left(\mathrm{H}_{3}\right) \mathrm{f}(\mathrm{x}, \mathrm{u})=\mathrm{o}(\mathrm{u}) \mathrm{u} \rightarrow 0$ uniformly in $x \in \mathbb{R}^{3}$;

$\left(\mathrm{H}_{4}\right) \mathrm{f}(\mathrm{x}, \mathrm{u}) \mathrm{u}-4 \mathrm{~F}(\mathrm{x}, \mathrm{u}) \geqslant 0$ for all $(\mathrm{x}, \mathrm{u}) \in\left(\mathbb{R}^{3}, \mathbb{R}\right)$;

$\left(\mathrm{H}_{5}\right) \lim _{|\mathfrak{u}| \rightarrow \infty} \frac{\mathrm{F}(\mathrm{x}, \mathfrak{u})}{|\mathfrak{u}|^{4}}=+\infty$ uniformly in $x \in \mathbb{R}^{3}$;

$\left(\mathrm{H}_{6}\right)$ there exist $\mathrm{V}_{0}, \mathrm{~K}_{0} \in \mathrm{L}^{\infty}\left(\mathbb{R}^{3}\right), \mathrm{f}_{0} \in \mathrm{C}\left(\mathbb{R}^{3} \times \mathbb{R}, \mathbb{R}\right)$ satisfies:

(i) $V_{0}, K_{0}$, and $f_{0}$ are 1 -periodic in $x_{i}, 1 \leqslant i \leqslant 3$;

(ii) $V-V_{0}, K-K_{0} \in \mathfrak{I},\left|f(x, u)-f_{0}(x, u)\right| \leqslant|h(x)|\left(|u|+|u|^{p}\right), x \in \mathbb{R}^{3}, h \in \mathfrak{I}$;

(iii) $V \leqslant V_{0}, K \leqslant K_{0}, F(x, t) \geqslant F_{0}(x, t)=\int_{0}^{t} f_{0}(x, s) d s$ for all $(x, t) \in\left(\mathbb{R}^{3}, \mathbb{R}\right)$;

(iv) $\forall u \neq 0, s \mapsto \frac{f_{0}(x, s u)}{s^{3}}$ is nondecreasing on $(-\infty, 0)$ and $(0, \infty)$.

Our main results of this paper is as follows.

Theorem 1.1. Assume $\left(\mathrm{H}_{1}\right)-\left(\mathrm{H}_{6}\right)$ are satisfied, then system (1.7) has at least one solution.

Theorem 1.2. Suppose that $\mathrm{V}(\mathrm{x}), \mathrm{K}(\mathrm{x})$, and $\mathrm{f}(\mathrm{x}, \mathrm{t})$ are 1-periodic in $\mathrm{x}_{\mathrm{i}}, 1 \leqslant \mathrm{i} \leqslant 3$, and $\mathrm{V}(\mathrm{x}) \geqslant \mathrm{a}_{0}>0$ for all $x \in \mathbb{R}^{3}$. If $\mathrm{f}$ satisfies $\left(\mathrm{H}_{2}\right),\left(\mathrm{H}_{3}\right),\left(\mathrm{H}_{5}\right)$, and

$\left(\mathrm{H}_{4}\right)^{\star} \mathrm{f}(\mathrm{x}, \mathrm{u}) \mathrm{u}-4 \mathrm{~F}(\mathrm{x}, \mathrm{u})>0$ for all $\mathrm{u} \neq 0$,

then system (1.7) has a ground-state solution.

Remark 1.3.

(1) In this paper, the condition $\left(\mathrm{H}_{6}\right)$ means asymptotically periodic case of $V, K$, and $f$ at infinity. This condition was introduced by Lins and Silva [27] in the study of a Schrödinger equation. 
(2) In our paper, $f$ does not satisfy any monotone condition, that is $\frac{f(x, t)}{t}$ is oscillatory, and therefore the method of Nehari manifold [39] used in [45] is not applicable.

(3) In Theorem 1.1, in case of $\left(\mathrm{H}_{4}\right)$ being replaced by

$$
f(x, u) u-4 F(x, u) \geqslant-\sigma u^{2} \text { uniformly in } x \in \mathbb{R}^{3},
$$

where $0<\sigma<\inf _{\mathbb{R}^{3}} V$, then the result will still hold.

\section{Notation and preliminaries}

The scalar product and norm in Sobolev space $\mathrm{H}^{1}\left(\mathbb{R}^{3}\right)$ is defined by

$$
\langle\mathfrak{u}, v\rangle=\int_{\mathbb{R}^{3}}(\nabla \mathfrak{u} \cdot \nabla v+\mathrm{V}(\mathrm{x}) \mathrm{u} v) \mathrm{dx},\|\mathrm{u}\|^{2}=\langle\mathrm{u}, \mathrm{u}\rangle
$$

Set

$$
\|u\|_{0}^{2}=\int_{\mathbb{R}^{3}}\left(|\nabla u|+V_{0}(x) u^{2}\right) d x
$$

$\|\mathrm{u}\|_{0}$ is an equivalent norm in $\mathrm{H}^{1}\left(\mathbb{R}^{3}\right)$ since condition $\left(\mathrm{H}_{1}\right)$.

$D^{1,2}\left(\mathbb{R}^{3}\right)$ is the Sobolev space endowed with the scalar product and norm

$$
\langle\mathrm{u}, v\rangle_{\mathrm{D}^{1,2}}=\int_{\mathbb{R}^{3}} \nabla \mathfrak{u} \cdot \nabla v \mathrm{~d} x,\|\mathrm{u}\|_{\mathrm{D}^{1,2}}^{2}=\int_{\mathbb{R}^{3}}|\nabla \mathrm{u}|^{2} \mathrm{~d} x .
$$

Since $K \in \mathrm{L}^{\infty}\left(\mathbb{R}^{3}\right), \inf _{\mathbb{R}^{3}} \mathrm{~K}>0, \forall \mathrm{u} \in \mathrm{H}^{1}\left(\mathbb{R}^{3}\right)$, by Lax-Milgram theorem, there exists unique $\phi=\phi_{\mathfrak{u}} \in$ $\mathrm{D}^{1,2}\left(\mathbb{R}^{3}\right)$ such that

$$
-\Delta \phi=\mathrm{K}(\mathrm{x}) \mathrm{u}^{2} \text {. }
$$

Functional $\phi_{\mathfrak{u}}$ satisfies the following properties.

Lemma $2.1([9,11,32,45,46]) . \forall u \in \mathrm{H}^{1}\left(\mathbb{R}^{3}\right)$,

(i) there exists $C>0$ such that $\left\|\phi_{u}\right\|_{D^{1,2}} \leqslant C\|u\|^{2}$ and

$$
\int_{\mathbb{R}^{3}}\left|\nabla \phi_{\mathfrak{u}}\right|^{2} \mathrm{~d} x \leqslant \int_{\mathbb{R}^{3}} \mathrm{~K}(\mathrm{x}) \phi_{\mathfrak{u}} \mathrm{u}^{2} \mathrm{~d} x \leqslant \mathrm{C}\|\mathrm{u}\|^{4}, \forall \mathrm{u} \in \mathrm{H}^{1}\left(\mathbb{R}^{3}\right) ;
$$

(ii) $\phi_{\mathfrak{u}} \geqslant 0, \forall \mathrm{u} \in \mathrm{H}^{1}\left(\mathbb{R}^{3}\right)$;

(iii) $\phi_{\mathrm{tu}}=\mathrm{t}^{2} \phi_{\mathrm{u}}, \forall \mathrm{t}>0, \forall \mathrm{u} \in \mathrm{H}^{1}\left(\mathbb{R}^{3}\right)$;

(iv) If $\mathrm{u}_{\mathfrak{n}} \rightarrow \mathrm{u}$ in $\mathrm{H}^{1}\left(\mathbb{R}^{3}\right)$, then $\phi_{u_{n}} \rightarrow \phi_{\mathfrak{u}}$ in $\mathrm{D}^{1,2}\left(\mathbb{R}^{3}\right)$.

Lemma 2.2. Suppose that $\mathrm{f}$ satisfies $\left(\mathrm{H}_{2}\right)$ and $\left(\mathrm{H}_{3}\right)$. Then, for any given $\varepsilon>0$ there exist $\mathrm{C}_{\varepsilon}$ such that

$$
|\mathbf{f}(x, t)| \leqslant \varepsilon|t|+\left.C_{\varepsilon}|t|\right|^{p},|F(x, t)| \leqslant \varepsilon|t|^{2}+\left.C_{\varepsilon}|t|\right|^{p+1} \text { for all }(x, t) \in\left(\mathbb{R}^{3}, \mathbb{R}\right) .
$$

The energy functional $\mathrm{I}: \mathrm{H}^{1}\left(\mathbb{R}^{3}\right) \rightarrow \mathbb{R}$ corresponding to system (1.7) is defined by

$$
\mathrm{I}(\mathrm{u})=\frac{1}{2} \int_{\mathbb{R}^{3}}\left(|\nabla u|^{2}+\mathrm{V}(\mathrm{x}) \mathrm{u}^{2}\right) \mathrm{d} x+\frac{1}{4} \int_{\mathbb{R}^{3}} \mathrm{~K}(\mathrm{x}) \phi_{\mathfrak{u}} \mathrm{u}^{2} \mathrm{~d} x-\int_{\mathbb{R}^{3}} \mathrm{~F}(\mathrm{x}, \mathrm{u}) \mathrm{d} x .
$$

In fact,

$$
I(u)=\frac{1}{2}\|u\|^{2}+\frac{1}{4} \int_{\mathbb{R}^{3}} K(x) \phi u u^{2} d x-\int_{\mathbb{R}^{3}} F(x, u) d x .
$$

In view Lemma of 2.2, the functional I is well defined. Furthermore, under our condition, $\mathrm{I} \in \mathrm{C}^{1}\left(\mathrm{H}^{1}\left(\mathbb{R}^{3}\right)\right)$ and $(u, \phi) \in H^{1}\left(\mathbb{R}^{3}\right) \times D^{1,2}\left(\mathbb{R}^{3}\right)$ is a solution of system (1.7) if and only if $u \in H^{1}\left(\mathbb{R}^{3}\right)$ is a critical point of I and $\phi=\phi_{\mathfrak{u}}$. 
$\forall \mathrm{u} \in \mathrm{H}^{1}\left(\mathbb{R}^{3}\right)$, let $\tilde{\phi}_{\mathfrak{u}} \in \mathrm{D}^{1,2}\left(\mathbb{R}^{3}\right)$ is unique solution of the following equation

$$
-\Delta \phi=\mathrm{K}_{0}(\mathrm{x}) \mathrm{u}^{2}
$$

Then $I_{0}(u)=\frac{1}{2}\|u\|_{0}^{2}+\frac{1}{4} \int_{\mathbb{R}^{3}} K_{0}(x) \tilde{\phi}_{u} u^{2} d x-\int_{\mathbb{R}^{3}} F_{0}(x, u) d x$ is the energy functional corresponding to the following system

$$
\begin{cases}-\Delta u+V_{0}(x) u+K_{0}(x) \phi u=f_{0}(x, u), & x \in \mathbb{R}^{3}, \\ -\Delta \phi=K_{0}(x) u^{2}, & x \in \mathbb{R}^{3} .\end{cases}
$$

Lemma 2.3 ([45]). If (i) of $\left(\mathrm{H}_{6}\right)$ holds, then

$$
\mathrm{G}(\mathrm{u}(\cdot+\mathrm{y}))=\mathrm{G}(\mathrm{u}), \forall \mathrm{y} \in \mathbb{Z}^{3}, \mathrm{u} \in \mathrm{H}^{1}\left(\mathbb{R}^{3}\right),
$$

where $\mathrm{G}(\mathrm{u})=\int_{\mathbb{R}^{3}} \mathrm{~K}_{0}(\mathrm{x}) \tilde{\phi}_{\mathfrak{u}} \mathrm{u}^{2} \mathrm{~d} \mathrm{x}$.

Let $u_{n} \subset H^{1}\left(\mathbb{R}^{3}\right)$, we said $u_{n}$ is a Cerami sequence for the functional $I$ at level $c \in \mathbb{R}$ if

$$
\mathrm{I}\left(\mathrm{u}_{\mathrm{n}}\right) \rightarrow \mathrm{c},\left(1+\left\|\mathrm{u}_{\mathrm{n}}\right\|\right) \mathrm{I}^{\prime}\left(\mathrm{u}_{\mathrm{n}}\right) \rightarrow 0, \mathrm{n} \rightarrow \infty .
$$

The following result is a version of the classical mountain pass theorem [4, 43]. For the proof, please see [33].

Theorem 2.4. Let $\mathrm{E}$ be a real Banach space. Assume $\mathrm{I} \in \mathrm{C}^{\prime}(\mathrm{E}, \mathbb{R})$ satisfies $\mathrm{I}(0)=0$ and

( $\left.\mathrm{I}_{1}\right)$ there exist $\rho, \alpha>0$ such that $\mathrm{I}(\mathrm{u}) \geqslant \alpha>0$ for all $\|\mathrm{u}\|=\rho$;

$\left(\mathrm{I}_{2}\right)$ there exist $\mathrm{e} \in \mathrm{E}$ with $\|\mathrm{e}\|>\rho$ such that $\mathrm{I}(\mathrm{e}) \leqslant 0$.

Then I possesses a Cerami sequence at level

$$
c=\inf _{\Theta} \max _{t \in[0,1]} I(\gamma(t))
$$

where

$$
\Theta=\gamma \in \mathrm{C}([0,1], \mathrm{E}): \gamma(0)=0,\|\gamma(1)\|>\rho, \mathrm{I}(\gamma(1)) \leqslant 0 .
$$

Theorem 2.5 (local mountain pass theorem [27]). Let $\mathrm{E}$ be a real Banach space. Assume $\mathrm{I} \in \mathrm{C}^{\prime}(\mathrm{E}, \mathbb{R})$ satisfies $\mathrm{I}(0)=0,\left(\mathrm{I}_{1}\right)$ and $\left(\mathrm{I}_{2}\right)$. If there exists $\gamma_{0} \in \Theta, \Theta$ defined as in Theorem 2.4, such that

$$
c=\max _{t \in[0,1]} I\left(\gamma_{0}(t)\right)>0,
$$

then I possesses a non-trivial critical point $u \in \gamma_{0}([0,1])$ at the level c.

Lemma 2.6. Suppose that $\mathrm{f}$ satisfies $\left(\mathrm{H}_{1}\right),\left(\mathrm{H}_{2}\right),\left(\mathrm{H}_{3}\right)$, and $\left(\mathrm{H}_{5}\right)$. Then $\mathrm{I}$ satisfies $\left(\mathrm{I}_{1}\right)$ and $\left(\mathrm{I}_{2}\right)$.

Proof. By Lemma 2.2 and Sobolev's inequality, we have

$$
\int_{\mathbb{R}^{N}} F(x, u) d x \leqslant \varepsilon|u|_{2}^{2}+C_{\varepsilon}|u|_{p+1}^{p+1} \leqslant \varepsilon C_{1}\|u\|^{2}+C\|u\|^{p+1}
$$

for some $C_{1}>0$. By $\int_{\mathbb{R}^{3}} K(x) \phi_{u} u^{2} d x \geqslant 0$, we have

$$
\mathrm{I}(\mathrm{u}) \geqslant \frac{1}{2}\|\mathrm{u}\|^{2}-\mathrm{C}_{1} \varepsilon\|\mathrm{u}\|^{2}-\mathrm{C}\|\mathrm{u}\|^{\mathrm{p}+1}=\left(\frac{1}{2}-\mathrm{C}_{1} \varepsilon\right)\|\mathrm{u}\|^{2}-\mathrm{C}\|\mathrm{u}\|^{\mathrm{p}+1} .
$$

Since $p>2$, we have

$$
\mathrm{I}(\mathrm{u}) \geqslant\left(\frac{1}{2}-\mathrm{C}_{1} \varepsilon\right)\|\mathrm{u}\|^{2}+\mathrm{o}\left(\|\mathrm{u}\|^{\mathrm{p}}\right) \geqslant \alpha
$$


for $\|u\|=\rho$ small enough. This proves $\left(\mathrm{I}_{1}\right)$.

Next we prove $\exists e \in \mathrm{H}^{1}\left(\mathbb{R}^{3}\right)$ such that $\mathrm{I}(e)<0$. By $\left(\mathrm{H}_{3}\right)$ and $\left(\mathrm{H}_{5}\right)$, for any $0 \neq v \in \mathrm{H}^{1}\left(\mathbb{R}^{3}\right)$ that satisfies

$$
M \int_{\mathbb{R}^{3}} v^{4} \mathrm{~d} x>\frac{1}{4} \int_{\mathbb{R}^{3}} \mathrm{~K}(x) \phi_{\nu} v^{2} \mathrm{~d} x
$$

there exists $C>0$ such that

$$
\mathrm{F}(\mathrm{x}, \mathrm{u}) \geqslant M \mathrm{u}^{4}-\mathrm{Cu} \mathrm{u}^{2}, \quad \forall(\mathrm{x}, \mathrm{u}) \in \mathbb{R}^{3} \times \mathbb{R} .
$$

Hence

$$
\begin{aligned}
\mathrm{I}(\mathrm{t} v) & =\frac{\mathrm{t}^{2}}{2}\|v\|^{2}+\frac{\mathrm{t}^{4}}{4} \int_{\mathbb{R}^{3}} \mathrm{~K}(\mathrm{x}) \phi_{v} v^{2} \mathrm{~d} x-\int_{\mathbb{R}^{3}} \mathrm{~F}(\mathrm{x}, \mathrm{t} v) \mathrm{d} x \\
& \leqslant \frac{\mathrm{t}^{2}}{2}\|v\|^{2}+\frac{\mathrm{t}^{4}}{4} \int_{\mathbb{R}^{3}} \mathrm{~K}(\mathrm{x}) \phi_{v} v^{2} \mathrm{~d} x-M \mathrm{t}^{4} \int_{\mathbb{R}^{3}} v^{4} \mathrm{~d} x+C \mathrm{t}^{2} \int_{\mathbb{R}^{3}} v^{2} \mathrm{~d} x \\
& =\left(\mathrm{C}+\frac{1}{2}\right) \mathrm{t}^{2}\|v\|^{2}-\left(\mathrm{M} \int_{\mathbb{R}^{3}} v^{4} \mathrm{~d} x-\frac{1}{4} \int_{\mathbb{R}^{3}} \mathrm{~K}(x) \phi_{v} v^{2} \mathrm{~d} x\right) \mathrm{t}^{4} \\
& \rightarrow-\infty
\end{aligned}
$$

as $t \rightarrow \infty$. So, for $t$ sufficient large, choose $e=t v$.

Lemma 2.7. Suppose that $\mathrm{f}$ satisfies $\left(\mathrm{H}_{1}\right)-\left(\mathrm{H}_{5}\right)$. Then any Cerami sequence for $\mathrm{I}$ is bounded.

Proof. Let $\mathrm{u}_{\mathrm{n}} \subset \mathrm{H}^{1}\left(\mathbb{R}^{3}\right)$ be such that

$$
\mathrm{I}\left(\mathrm{u}_{\mathrm{n}}\right) \rightarrow \mathrm{c},\left(1+\left\|\mathrm{u}_{\mathrm{n}}\right\|\right) \mathrm{I}^{\prime}\left(\mathrm{u}_{\mathrm{n}}\right) \rightarrow 0, \mathrm{n} \rightarrow \infty
$$

Since

$$
c+o_{n}(1)=4 I\left(u_{n}\right)-I^{\prime}\left(u_{n}\right) u_{n}=\left\|u_{n}\right\|^{2}+\int_{\mathbb{R}^{3}}\left(f\left(x, u_{n}\right) u_{n}-4 F\left(x, u_{n}\right)\right) d x \geqslant\left\|u_{n}\right\|^{2} .
$$

From above inequality, $u_{n}$ is bounded.

Lemma 2.8. Suppose that $\mathrm{f}$ satisfies $\left(\mathrm{H}_{1}\right)-\left(\mathrm{H}_{5}\right)$. Let $\mathrm{u}_{\mathrm{n}} \subset \mathrm{H}^{1}\left(\mathbb{R}^{3}\right)$ be Cerami sequence for $\mathrm{I}$ at level $\mathrm{c}>0$. If $u_{n} \rightarrow 0$ in $\mathrm{H}^{1}\left(\mathbb{R}^{3}\right)$, then there exist a sequence $\left\{y_{n}\right\} \subset \mathbb{R}^{3}$ and $\mathrm{R}>0, \beta>0$ such that $\mathrm{y}_{\mathrm{n}} \rightarrow \infty$ and

$$
\lim _{n \rightarrow \infty} \sup \int_{B_{R}\left(y_{n}\right)}\left|u_{n}\right|^{2} \geqslant \beta>0
$$

Proof. Suppose by contradiction, that the Lemma fails. Then, for any $\mathrm{R}>0$, we have that

$$
\lim _{n \rightarrow \infty} \sup \int_{B_{R}(y)}\left|u_{n}\right|^{2}=0
$$

for all $R>0$. By Lions Lemma [43], we have that $\left|u_{n}\right|_{L^{s}} \rightarrow 0$ for any $s \in\left(2,2^{*}\right)$.

By Lemma 2.2, we have $\int_{\mathbb{R}^{3}} f\left(x, u_{n}\right) u_{n} \rightarrow 0$.

Since $\mathrm{I}^{\prime}\left(u_{n}\right) u_{n} \rightarrow 0$ as $n \rightarrow \infty$, we get

$$
\left\|u_{n}\right\|^{2} \leqslant\left\|u_{n}\right\|^{2}+\int_{\mathbb{R}^{3}} K(x) \phi_{u_{n}} u_{n}^{2} d x=\int_{\mathbb{R}^{3}} f\left(x, u_{n}\right) u_{n} d x+o_{n}(1) .
$$

So, $u_{n} \rightarrow 0$ in $H^{1}\left(\mathbb{R}^{3}\right)$. Therefore, $\int_{\mathbb{R}^{3}} K(x) \phi_{u_{n}} u_{n}^{2} d x \rightarrow 0$.

From above facts, we get $\mathrm{I}\left(\mathrm{u}_{n}\right) \rightarrow 0$ as $n \rightarrow \infty$, which contradicts with $\mathrm{I}\left(\mathrm{u}_{n}\right) \rightarrow c>0$. 
Lemma 2.9 ([45]). Suppose that (ii) of $\left(\mathrm{H}_{6}\right)$ holds. If $\left\{\mathrm{u}_{\mathrm{n}}\right\} \in \mathrm{H}^{1}\left(\mathbb{R}^{3}\right)$ such that $\mathrm{u}_{\mathrm{n}} \rightarrow 0$ in $\mathrm{H}^{1}\left(\mathbb{R}^{3}\right),\left\{\varphi_{n}\right\} \in \mathrm{H}^{1}\left(\mathbb{R}^{3}\right)$ is bounded, then

$$
\begin{aligned}
& \int_{\mathbb{R}^{3}}\left[\mathrm{~V}(x)-\mathrm{V}_{0}(x)\right] \mathrm{u}_{n} \varphi_{n} \mathrm{~d} x \rightarrow 0, \\
& \int_{\mathbb{R}^{3}}\left[\mathrm{~K}(x) \phi_{u_{n}} u_{n} \varphi_{n}-K_{0}(x) \tilde{\phi}_{u_{n}} u_{n} \varphi_{n}\right] d x \rightarrow 0, \\
& \int_{\mathbb{R}^{3}}\left[f\left(x, u_{n}\right)-f_{0}\left(x, u_{n}\right)\right] \varphi_{n} d x \rightarrow 0 .
\end{aligned}
$$

\section{Proof of main result}

In this section we are ready to prove our main theorems.

Proof of Theorem 1.1. In view of Lemma 2.6 and Theorem 2.4, there exists a sequence $\left(u_{n}\right) \subset H^{1}\left(\mathbb{R}^{3}\right)$ such that

$$
\mathrm{I}^{\prime}\left(\mathrm{u}_{\mathrm{n}}\right) \rightarrow \mathrm{c} \geqslant \alpha>0 \text { and }\left(1+\left\|\mathrm{u}_{\mathrm{n}}\right\|\right) \mathrm{I}^{\prime}\left(\mathrm{u}_{\mathrm{n}}\right) \rightarrow 0 \text { as } \mathrm{n} \rightarrow \infty .
$$

From Lemma 2.7, $\left\{u_{n}\right\}$ is bounded. So, without loss of generality, one assumes that $u_{n} \rightarrow \mathfrak{u}$ weakly in $\mathrm{H}^{1}\left(\mathbb{R}^{3}\right)$.

Now we prove $\mathrm{I}^{\prime}(\mathfrak{u})=0$. Indeed, since $\mathrm{C}_{0}^{\infty}\left(\mathbb{R}^{3}\right)$ is dense in $\mathrm{H}^{1}\left(\mathbb{R}^{3}\right)$, it suffices to show that $\mathrm{I}^{\prime}(u) \varphi=0$ for all $\varphi \in \mathrm{C}_{0}^{\infty}\left(\mathbb{R}^{3}\right) . \forall \varphi \in \mathrm{C}_{0}^{\infty}\left(\mathbb{R}^{3}\right)$, we have

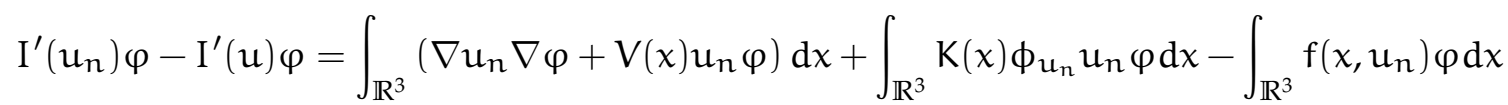

$$
\begin{aligned}
& -\int_{\mathbb{R}^{3}}(\nabla u \nabla \varphi+V(x) u \varphi) d x-\int_{\mathbb{R}^{3}} K(x) \phi_{u} u \varphi d x+\int_{\mathbb{R}^{3}} f(x, u) \varphi d x \\
& =\left\langle u_{n}-\mathfrak{u}, \varphi\right\rangle-\int_{\mathbb{R}^{3}} K(x)\left(\phi_{\mathfrak{u}_{n}} u_{n}-\phi_{\mathfrak{u}} u\right) \varphi d x \\
& -\int_{\mathbb{R}^{3}}\left(f\left(x, u_{n}\right)-f(x, u)\right) \varphi d x .
\end{aligned}
$$

Since $u_{n} \rightarrow u$, by Lemmas 2.1 and 2.2, we obtain

$$
\mathrm{I}^{\prime}(\mathrm{u}) \varphi=\lim _{\mathrm{n} \rightarrow \infty} \mathrm{I}^{\prime}\left(\mathrm{u}_{\mathrm{n}}\right) \varphi=0,
$$

which implies that $\mathrm{I}^{\prime}(\mathrm{u})=0$.

If $u \neq 0$, the theorem is proved.

If $u=0$, from Lemma 2.8 , there exists a sequence $\left(y_{n}\right) \subset \mathbb{R}^{3}, R>0, \beta>0$ such that $\left|y_{n}\right| \rightarrow \infty$ as $n \rightarrow \infty$ and

$$
\limsup _{n \rightarrow \infty} \int_{B_{R(y n)}}\left|u_{n}\right|^{2} \geqslant \beta>0 .
$$

Let $\left(y_{n}\right) \subset \mathbb{Z}^{3}$ and $\tilde{u}_{n}(x)=u_{n}\left(x+y_{n}\right)$, and observing that $\left\|\tilde{u}_{n}\right\|=\left\|u_{n}\right\|_{0}$, up to a subsequence we have that $\tilde{u}_{n} \rightarrow \tilde{u}$ in $H^{1}\left(\mathbb{R}^{3}\right), \tilde{u}_{n} \rightarrow \tilde{u}$ in $L_{\text {loc }}^{2}\left(\mathbb{R}^{3}\right)$ and for almost every $x \in \mathbb{R}^{3}$. From (3.2), we have $\tilde{u} \neq 0$.

Next we prove $I_{0}^{\prime}(\tilde{u})=0 . \forall \varphi \in C_{0}^{\infty}\left(\mathbb{R}^{3}\right)$, for each $n \in \mathbb{N}$, let $\varphi_{n}(x)=\varphi\left(x-y_{n}\right)$, we get that

$$
\mathrm{I}_{0}^{\prime}(\tilde{\mathrm{u}}) \varphi=\mathrm{I}_{0}^{\prime}\left(\tilde{\mathrm{u}}_{\mathfrak{n}}\right) \varphi+\mathrm{o}_{\mathfrak{n}}(1)=\mathrm{I}_{0}^{\prime}\left(\mathrm{u}_{\mathfrak{n}}\right) \varphi_{\mathfrak{n}}+\mathrm{o}_{\mathfrak{n}}(1) .
$$

On the other hand, by Lemma 2.9 , we get that

$$
\begin{aligned}
& \mathrm{I}_{0}^{\prime}\left(\mathrm{u}_{\mathrm{n}}\right) \varphi_{\mathrm{n}}=\mathrm{I}^{\prime}\left(\mathrm{u}_{\mathrm{n}}\right) \varphi_{\mathrm{n}}+\int_{\mathbb{R}^{3}}\left[\mathrm{~V}_{0}(\mathrm{x})-\mathrm{V}(\mathrm{x})\right] \mathrm{u}_{\mathrm{n}} \varphi_{\mathrm{n}} \mathrm{d} \mathrm{x} \\
& -\int_{\mathbb{R}^{3}}\left[f_{0}\left(x, u_{n}\right)-f(x, u)\right] \varphi_{n} d x-\int_{\mathbb{R}^{3}}\left[K(x) \phi_{u_{n}} u_{n} \varphi_{n}-K_{0}(x) \tilde{\phi}_{u_{n}} u_{n} \varphi_{n}\right] d x \\
& =\mathrm{I}^{\prime}\left(\mathrm{u}_{\mathrm{n}}\right) \varphi_{\mathrm{n}}+\mathrm{o}_{\mathrm{n}}(1) \text {. }
\end{aligned}
$$


So, by (3.1), we get $\mathrm{I}_{0}^{\prime}(\tilde{\mathrm{u}})=0$.

By Lemma 2.9, similar to above, we have

$$
\mathrm{I}\left(\mathrm{u}_{\mathrm{n}}\right)-\mathrm{I}_{0}\left(\mathrm{u}_{n}\right) \rightarrow 0, \quad \mathrm{I}^{\prime}\left(\mathrm{u}_{\mathrm{n}}\right) \mathrm{u}_{\mathrm{n}}-\mathrm{I}_{0}^{\prime}\left(\mathrm{u}_{n}\right) \mathrm{u}_{\mathrm{n}} \rightarrow 0 .
$$

Then

$$
\mathrm{I}_{0}\left(\mathrm{u}_{\mathrm{n}}\right) \rightarrow \mathrm{c}, \quad \mathrm{I}_{0}^{\prime}\left(\mathrm{u}_{\mathrm{n}}\right) \mathrm{u}_{\mathrm{n}} \rightarrow 0 .
$$

By (iv) of $\left(\mathrm{H}_{6}\right), \forall u \in \mathbb{R}$, we have $4 \mathrm{~F}_{0}(\mathrm{x}, \mathrm{u}) \leqslant \mathrm{f}_{0}(\mathrm{x}, \mathrm{u})$. So

$$
\begin{aligned}
c+o_{n}(1) & =I_{0}\left(u_{n}\right)-\frac{1}{4} I_{0}^{\prime}\left(u_{n}\right) u_{n} \\
& =\frac{1}{4}\left\|u_{n}\right\|_{0}^{2}+\int_{\mathbb{R}^{3}}\left[\frac{1}{4} f_{0}\left(x, u_{n}\right) u_{n}-F_{0}\left(x, u_{n}\right)\right] d x \\
& =\frac{1}{4}\left\|\tilde{u}_{n}\right\|_{0}^{2}+\int_{\mathbb{R}^{3}}\left[\frac{1}{4} f_{0}\left(x, \tilde{u}_{n}\right) \tilde{u}_{n}-F_{0}\left(x, \tilde{u}_{n}\right)\right] d x \\
& \geqslant \frac{1}{4}\|\tilde{u}\|_{0}^{2}+\int_{\mathbb{R}^{3}}\left[\frac{1}{4} f_{0}(x, \tilde{u}) \tilde{u}-F_{0}(x, \tilde{u})\right] d x+o_{n}(1) \\
& =I_{0}(\tilde{u})-\frac{1}{4} I_{0}^{\prime}(\tilde{u}) \tilde{u}+o_{n}(1) \\
& =I_{0}(\tilde{u})+o_{n}(1) .
\end{aligned}
$$

Therefore $\mathrm{I}_{0}(\tilde{\mathrm{u}}) \leqslant \mathrm{c}$.

We shall verify that $\max _{\mathrm{t} \geqslant 0} \mathrm{I}_{0}(\mathrm{t} \tilde{\mathrm{u}})=\mathrm{I}_{0}(\tilde{\mathrm{u}})$. Let

$$
\chi(t)=I_{0}(t \tilde{u})=\frac{t^{2}}{2}\|\tilde{u}\|_{0}^{2}+\frac{t^{4}}{4} \int_{\mathbb{R}^{3}} K_{0}(x) \phi_{\tilde{u}} \tilde{u}^{2} d x-\int_{\mathbb{R}^{3}} F_{0}(x, t \tilde{u}) d x .
$$

So,

$$
\begin{aligned}
x^{\prime}(t) & =t\|\tilde{u}\|_{0}^{2}+t^{3} \int_{\mathbb{R}^{3}} K_{0}(x) \phi_{\tilde{u}} \tilde{u}^{2} d x-\int_{\mathbb{R}^{3}} f_{0}(x, t \tilde{u}) \tilde{u} d x \\
& =t^{3}\left(\frac{1}{t^{2}}\|\tilde{u}\|_{0}^{2}+\int_{\mathbb{R}^{3}} K_{0}(x) \phi_{\tilde{u}} \tilde{u}^{2} d x-\int_{\mathbb{R}^{3}} \frac{f_{0}(x, t \tilde{u}) \tilde{u}}{t^{3}}\right) d x=t^{3} A(t) .
\end{aligned}
$$

Since $I_{0}^{\prime}(\tilde{u})=0, A(1)=0$. It follows from part (iv) of $\left(H_{6}\right)$ that $A$ is strictly decreasing in $(0, \infty)$, then $A(t)>0$ when $t \in(0,1)$ and $A(t)<0$ when $t \in(1, \infty)$. Therefore

$$
\chi^{\prime}(t)>0 \text { when } t \in(0,1) \text { and } \chi^{\prime}(t)<0 \text { when } t \in(1, \infty) .
$$

Hence, $\max _{\mathrm{t} \geqslant 0} \mathrm{I}_{0}(\mathrm{t} \tilde{\mathrm{u}})=\mathrm{I}_{0}(\tilde{\mathrm{u}})$.

By the definition of $c,(V)$ and part (iii) of $\left(\mathrm{H}_{6}\right)$, we have that

$$
c \leqslant \max _{t \geqslant 0} I(t \tilde{u}) \leqslant \max _{t \geqslant 0} I_{0}(t \tilde{u})=I_{0}(\tilde{u}) \leqslant c .
$$

We can now invoke Theorem 2.5 to conclude that I possesses a critical point at level $c>0$. This finishes the proof.

Proof of Theorem 1.2. It is easy to see that Lemmas 2.2, 2.6, 2.7, and 2.8 are all hold by using the conditions of Theorem 1.1. From Lemma 2.6 and Theorem 2.4, there exists Cerami sequence $\left\{u_{n}\right\} \subset H^{1}\left(\mathbb{R}^{3}\right)$, i.e.,

$$
\mathrm{I}_{0}\left(\mathrm{u}_{\mathrm{n}}\right) \rightarrow \mathrm{c}_{0} \text { and }\left(1+\left\|\mathrm{u}_{\mathrm{n}}\right\|_{0}\right) \mathrm{I}_{0}^{\prime}\left(\mathrm{u}_{\mathrm{n}}\right) \rightarrow 0, \text { as } \mathrm{n} \rightarrow+\infty
$$

where $c_{0}$ is the mountain pass level of $\mathrm{I}_{0}$. 
By Lemmas 2.7, we conclude that $u_{n} \rightarrow u$ weakly in $\mathrm{H}^{1}\left(\mathbb{R}^{3}\right)$. Similar to proof of Theorem 1.1, we have $\mathrm{I}_{0}^{\prime}(\mathrm{u})=0$.

Following, we only need to consider the case in which $u=0$. By Lemma 2.8, there is a sequence $\left(y_{n}\right) \subset \mathbb{Z}^{3}, R>0, \beta>0$ such that $\left|y_{n}\right| \rightarrow \infty$ as $n \rightarrow \infty$ and

$$
\limsup _{n \rightarrow \infty} \int_{B_{R\left(y_{n}\right)}}\left|u_{n}\right|^{2} \geqslant \beta>0 .
$$

Let $\tilde{u}_{n}(x)=u_{n}\left(x+y_{n}\right)$, then $\left\|\tilde{u}_{n}\right\|_{0}=\left\|u_{n}\right\|_{0}$. Up to a subsequence, we have

$$
\tilde{u}_{n} \rightarrow \tilde{u} \text { weakly in } H^{1}\left(\mathbb{R}^{3}\right), \quad \tilde{u}_{n} \rightarrow \tilde{u} \text { in } L_{l o c}^{2}\left(\mathbb{R}^{3}\right), \quad \tilde{u}_{n}(x) \rightarrow \tilde{u} \text { almost everywhere in } \mathbb{R}^{3} \text {. }
$$

By (3.3), $\tilde{u} \neq 0$. Similar to proof of Theorem 1.1 , we get $I_{0}^{\prime}(\tilde{u})=0$.

So $m=\inf \left\{I_{0}(u): u \in H^{1}\left(\mathbb{R}^{3}\right), I^{\prime}(u)=0\right\}>0$ is well defined. Next, to prove $m$ is achieved. Indeed, let $\left\{u_{n}\right\} \subset H^{1}\left(\mathbb{R}^{3}\right)$ be a minimizing sequence for $m$, i.e.,

$$
\mathrm{I}_{0}\left(\mathrm{u}_{\mathrm{n}}\right) \rightarrow \mathrm{m}, \quad \mathrm{I}_{0}^{\prime}\left(\mathrm{u}_{\mathrm{n}}\right)=0 \text { and } \mathrm{u}_{\mathrm{n}} \neq 0
$$

Obviously, $\left\{u_{n}\right\}$ is a Cerami sequence for $I_{0}$. So, from Lemma 2.7, $\left\{u_{n}\right\}$ is bounded. Moreover, from $I_{0}^{\prime}\left(u_{n}\right) u_{n}=0$ and Lemma 2.2, there exists $\sigma>0$ such that $\left\|u_{n}\right\|_{0} \geqslant \sigma$. Thus, arguing as in the preceding paragraph, we obtain a translated subsequence $\left\{\tilde{u}_{n}\right\}$, which has a non-zero weak limit $u_{0}$ such that $\mathrm{I}_{0}^{\prime}\left(\mathfrak{u}_{0}\right)=0$ and $\tilde{u}_{n}(x) \rightarrow \mathfrak{u}_{0}(x)$ a.e. in $\mathbb{R}^{\mathrm{N}}$. By Fatou's lemma

$$
\begin{aligned}
m=\lim _{n \rightarrow \infty} I_{0}\left(u_{n}\right)=\lim _{n \rightarrow \infty} I_{0}\left(\tilde{u}_{n}\right) & =\liminf _{n \rightarrow \infty} \frac{\left\|\tilde{u}_{n}\right\|_{0}}{4}+\liminf _{n \rightarrow \infty} \int_{\mathbb{R}^{3}} \hat{F}_{0}\left(x, \tilde{u}_{n}\right) d x \\
& \geqslant \frac{\left\|u_{0}\right\|_{0}}{4}+\int_{\mathbb{R}^{3}} \hat{F}_{0}\left(x, u_{0}\right) d x=I_{0}\left(u_{0}\right) .
\end{aligned}
$$

Consequently, $I_{0}\left(u_{0}\right)=m$, and therefore $u_{0} \neq 0$ is a ground-state solution.

\section{Acknowledgment}

The authors express their sincere thanks to the reviewers and editor for the useful suggestions to improve the paper.

\section{References}

[1] C. O. Alves, M. A. S. Souto, Existence of least energy nodal solution for a Schrödinger-Poisson system in bounded domains, Z. Angew. Math. Phys., 65 (2014), 1153-1166. 1

[2] C. O. Alves, M. A. S. Souto, S. H. M. Soares, Schrödinger-Poisson equations without Ambrosetti-Rabinowitz condition, J. Math. Anal. Appl., 377 (2011), 584-592. 1, 1, 1

[3] A. Ambrosetti, M. Badiale, S. Cingolani, Semiclassical states of nonlinear Schrödinger equations, Arch. Rational Mech. Anal., 140 (1997), 285-300. 1

[4] A. Ambrosetti, P. H. Rabinowitz, Dual variational methods in critical point theory and applications, J. Functional Analysis, 14 (1973), 349-381. 2

[5] A. Ambrosetti, D. Ruiz, Multiple bound states for the Schrödinger-Poisson equation, Commun. Contemp. Math., 10 (2008), 391-404. 1, 1

[6] A. Azzollini, Concentration and compactness in nonlinear Schrödinger-Poisson systemwith a general nonlinearity, J. Differential Equations, 249 (2010), 1746-1763.

[7] V. Benci, D. Fortunato, An eigenvalue problem for the Schrödinger-Maxwell equations, Topol. Methods Nonlinear Anal., 11 (1998), 283-293. 1

[8] H. Berestycki, P.-L. Lions, Nonlinear scalar field equations. II. Existence of infinitely many solutions, Arch. Rational Mech. Anal., 82 (1983), 347-375. 1

[9] G. Cerami, G. Vaira, Positive solutions for some non-autonomous Schrödinger-Poisson systems, J. Differential Equations, 248 (2010), 521-543. 1, 1, 2.1 
[10] G. M. Coclite, A multiplicity result for the nonlinear Schrödinger-Maxwell equations, Commun. Appl. Anal., 7 (2003), 417-423. 1

[11] T. D'Aprile, D. Mugnai, Solitary waves for nonlinear Klein-Gordon-Maxwell and Schrödinger-Maxwell equations, Proc. Roy. Soc. London Ser. A, 134 (2004), 893-906. 1, 2.1

[12] T. D'Aprile, D. Mugnai, Non-existence results for the coupled Klein-Gordon-Maxwell equations, Adv. Nonlinear Stud., 4 (2004), 307-322. 1

[13] T. D'Aprile, J. Wei, On bound states concentrating on spheres for the Maxwell-Schrödinger equation, SIAM J. Math. Anal., 37 (2005), 321-342. 1, 1

[14] R. de Marchi, Schrödinger equations with asymptotically periodic terms, Proc. Roy. Soc. Edinburgh Sect. A, 145 (2015), 745-757. 1

[15] M. del Pino, P. Felmer, Semi-classical states of nonlinear Schrödinger equations: A variational reduction method, Math. Ann., 324 (2002), 1-32.

[16] Y. Ding, F. Lin, Solutions of perturbed Schrödinger equations with critical nonlinearity, Calc. Var. Partial Differential Equations, 30 (2007), 231-249. 1

[17] X. He, W. Zou, Existence and concentration of ground states for Schrödinger-Poisson equations with critical growth, J. Math. Phys., 2012 (2012), 19 pages. 1

[18] L. Huang, E. M. Rocha, J. Chen, Two positive solutions of a class of Schrödinger-Poisson system with indefinite nonlinearity, J. Differential Equations, 255 (2013), 2463-2483.

[19] I. Ianni, Sign-changing radial solutions for the Schrödinger-Poisson-Slater problem, Topol. Methods Nonlinear Anal., 41 (2013), 365-385. 1

[20] L. Jeanjean, K. Tanaka, Singularly perturbed elliptic problems with superlinear or asympotically linear nonlinearities, Calc. Var. Partial Differential Equations, 21 (2004), 287-318. 1

[21] Y. Jiang, H.-S. Zhou, Schrödinger-Poisson system with steep potential well, J. Differential Equations, 251 (2011), $582-$ 608. 1

[22] S. Kim, J. Seok, On nodal solutions of the nonlinear Schrödinger-Poisson equations, Commun. Contemp. Math., 2012 (2012), 16 pages.

[23] G. Li, S. Peng, C. Wang, Multi-bump solutions for the nonlinear Schrödinger-Poisson system, J. Math. Phys., 2011 (2011), 19 pages. 1

[24] G. Li, S. Peng, S. Yan, Infinitely many positive solutions for the nonlinear Schrödinger-Poisson system, Commun. Contemp. Math., 12 (2010), 1069-1092. 1, 1

[25] G. Li, A. Szulkin, An asymptotically periodic Schrödinger equation with indefinite linear part, Commun. Contemp. Math., 4 (2002), 763-776. 1

[26] Y. Li, Z.-Q. Wang, J. Zeng, Ground states of nonlinear Schrödinger equations with potentials, Ann. Inst. H. Poincaré Anal. Non Linéaire, 23 (2006), 829-837. 1

[27] H. F. Lins, E. A. B. Silva, Quasilinear asymptotically periodic elliptic equations with critical growth, Nonlinear Anal., 71 (2009), 2890-2905. 1, 1, 2.5

[28] Z. Liu, S. Guo, Y. Fang, Multiple semiclassical states for coupled Schrödinger-Poisson equations with critical exponential growth, J. Math. Phys., 2015 (2015), 22 pages. 1

[29] Z. Liu, Z.-Q. Wang, J. Zhang, Infinitely many sign-changing solutions for the nonlinear Schrödinger-Poisson system, Ann. Mat. Pura Appl., 195 (2016), 775-794. 1

[30] P. H. Rabinowitz, Minimax theorems in critical point theory with applications to differential equations, Published for the Conference Board of the Mathematical Sciences, Washington, DC; by the American Mathematical Society, Providence, (1986). 1

[31] P. H. Rabinowitz, On a class of nonlinear Schrödinger equations, Z. Angew. Math. Phys., 43 (1992), 270-291. 1

[32] D. Ruiz, The Schrödinger-Poisson equation under the effect of a nonlinear local term, J. Funct. Anal., 237 (2006), 655-674. $1,2.1$

[33] M. Schechter, A variation of the mountain pass lemma and applications, J. London Math. Soc., 44 (1991), 491-502. 2

[34] W. Shuai, Q. Wang, Existence and asymptotic behavior of sign-changing solutions for the nonlinear Schrödinger-Poisson system in $\mathbb{R}^{3}$, Z. Angew. Math. Phys., 66 (2015), 3267-3282. 1

[35] E. A. B. Silva, G. F. Vieira, Quasilinear asymptotically periodic Schrödinger equations with subcritical growth, Nonlinear Anal., 72 (2010), 2935-2949. 1

[36] E. A. B. Silva, G. F. Vieira, Quasilinear asymptotically periodic Schrödinger equations with critical growth, Calc. Var. Partial Differential Equations, 39 (2010), 1-33. 1

[37] J. Sun, S. Ma, Ground state solutions for some Schrödinger-Poisson systems with periodic potentials, J. Differential Equations, 260 (2016), 2119-2149. 1

[38] J. Sun, T.-F. Wu, Z. Feng, Multiplicity of positive solutions for a nonlinear Schrödinger-Poisson system, J. Differential Equations, 260 (2016), 586-627. 1

[39] A. Szulkin, T. Weth, The method of Nehari manifold, Handbook of nonconvex analysis and applications, 597-632, Int. Press, Somerville, MA, (2010). 2

[40] G. Vaira, Gound states for Schrödinger-Poisson type systems, Ricerche di Matematica, 60 (2011), 263-297. 1, 1

[41] J. Wang, J. Xu, F. Zhang, X. Chen, Existence of multi-bump solutions for a semilinear Schrödinger-Poisson system, Nonlinearity, 26 (2013), 1377-1399. 
[42] Z. Wang, H.-S. Zhou, Sign-changing solutions for the nonlinear Schrödinger-Poisson system in, Calc. Var. Partial Differential Equations, 52 (2015), 927-943. 1

[43] M. Willem, Minimax Theorems, Birkhäuser Boston, Bosten, (1996). 2, 2

[44] M. Yang, F. Zhao, Y. Ding, On the existence of solutions for Schrödinger-Maxwell systems in $\mathbb{R}^{3}$, Rocky Mountain J. Math., 42 (2012), 1655-1674. 1

[45] H. Zhang, J. Xu, F. Zhang, Positive ground states for asymptotically periodic Schrödinger-Poisson systems, Math. Methods Appl. Sci., 36 (2013), 427-439. 1, 1, 2, 2.1, 2.3, 2.9

[46] L. Zhao, F. Zhao, On the existence of solutions for the Schrödinger-Poisson equations, J. Math. Anal. Appl., 346 (2008), 155-169. 1, 2.1 